\title{
Le peau à peau du nouveau-né en cours de césarienne : une étude d'évaluation des pratiques professionnelles.
}

Skin-to-skin during cesarean section : assessment of professional practices

Patricia LUU ${ }^{1}$, Béatrice MESTDAGH ${ }^{1}$, Catherine BARRE-DROUARD², Pierre RICHART $^{2}$, Clémence COURTECUISSE-VAMOUR ${ }^{1}$, Thameur RAKZA ${ }^{1}$, Charles GARABEDIAN $^{1,3}$, Damien SUBTIL ${ }^{1,4}$.

1) Univ. Lille, CHU Lille, Hôpital Jeanne de Flandre, F-59000 Lille, France

2) Univ. Lille, CHU Lille, Anesthésie Réanimation, F-59000 Lille, France

3) Univ. Lille, EA 4489 Environnement périnatal et croissance, F-59000 Lille France

4) Univ. Lille, EA 2694 Santé Publique, Epidémiologie et Qualité des Soins, F-59000 Lille France

\section{Corresponding author:}

Patricia LUU

Hôpital Jeanne de Flandre

Université Lille Nord de France

1 rue Eugène Avinée

59037 Lille Cedex, France

Tel: 33 +3 0320446626 - Fax: $33+30320446311$

e-mail: patricia_luu@yahoo.fr 
Titre court : Le peau à peau du nouveau-né en cours de césarienne (51 caractères) 


\section{Introduction}

A la naissance, le peau à peau du nouveau-né contre sa mère fait désormais partie des standards de la prise en charge néonatale [1-3]. D'abord pratiqué avec succès pour améliorer la survie et la qualité de vie des prématurés [3], le peau à peau est aujourd'hui recommandé pour tous les accouchements, quel que soit l'âge gestationnel [2]. II joue un rôle essentiel au sein du processus d'attachement, de plus en plus reconnu comme essentiel pour le développement cognitif ultérieur de l'enfant $[4,5]$.

Lors d'un accouchement par césarienne, la réalisation d'un peau à peau immédiat a longtemps été considérée comme très difficile ou impossible [6,7]. II est d'ailleurs probable que le retard de contact entre la mère et son enfant participe aux troubles constatés après une césarienne : instauration plus difficile du lien [8], diminution des taux d'allaitement maternel à la sortie [9], augmentation du risque de dépression du post-partum [8], insatisfaction maternelle à long terme [10].

Les études concernant la mise en place du peau à peau au bloc de césarienne, essentiellement américaines, ont été publiées à partir de 2008 [11-14]. Plusieurs équipes ont montré des effets très positifs pour le nouveau-né : maintien de la température [15], diminution du risque d'hypoglycémie [2], diminution de la douleur chez le nouveau-né [16]. En 2013, 59 \% des maternités aux Etats-Unis proposaient aux patientes d'initier le peau à peau en salle de césarienne [13]. 
Notre maternité ayant récemment tenté d'instaurer la réalisation d'un peau-à-peau quelle que soit la voie d'accouchement, nous avons décidé de mener une étude d'évaluation des pratiques professionnelles afin de savoir dans quelle mesure il était réellement possible de réaliser ce peau-à-peau en cours de césarienne, dès les premières minutes après le dégagement du nouveau-né. 


\section{Matériel et Méthodes}

II s'agit d'une étude descriptive et analytique menée dans une maternité de type 3 de type universitaire. Pour les accouchements par voie basse, le peau à peau y est systématique depuis 2012. Pour les césariennes en revanche, le peau à peau s'est installé de manière progressive depuis début 2015, moyennant quelques particularités d'organisation [17]: après clampage du cordon à une minute de vie, celui-ci est sectionné par l'opérateur. Le nouveau-né est confié à la sage-femme qui reçoit l'enfant dans un champ. Elle le place pendant une minute sur la table à langer située dans la salle, le sèche, prend sa température rectale et évalue son état. Si celui-ci est satisfaisant, elle se dirige vers la tête de la patiente - où se situent son conjoint et l'équipe d'anesthésie, et demande la permission aux parents et à l'équipe d'anesthésie de placer le nouveau-né sur le haut du thorax maternel (Figure 1). Idéalement, le passage préalable d'un bandeau de jersey autour du thorax maternel - enfilé comme une brassière juste avant la césarienne - permet de maintenir le nouveau-né « calé » transversalement contre sa mère.

Le nouveau-né n'est ensuite ôté du thorax maternel que si l'équipe d'anesthésie ou la maman le souhaitent, qu'il s'agisse d'un problème d'inconfort ou d'une nécessité médicale. II est alors confié au conjoint qui est invité à prendre le nouveau-né en peau à peau dans un fauteuil situé dans une pièce immédiatement adjacente (salle de réanimation néonatale). Les contre-indications au peau à peau maternel en cours de césarienne sont les mêmes que celles du peau à peau réalisé après un accouchement par voie basse (contre-indications néonatales : troubles d'adaptation à la vie extra-utérine, détresse respiratoire, pathologies et malformations graves du 
nouveau-né, mère traitée par médicaments dépresseurs respiratoires; contreindications maternelles: refus, état de conscience ou de disponibilité insuffisant, impossibilité matérielle pour l'équipe de surveiller la mère) [18].

Après deux années de pratique, l'ensemble des anesthésistes, des gynécologuesobstétriciens et des sages-femmes se sont dits favorables à cette mesure. Une étude d'évaluation des pratiques professionnelles a alors été jugée comme nécessaire pour évaluer à la fois la réalité et les obstacles au peau à peau en cas de césarienne.

Les patientes étaient éligibles si elles avaient accouché dans notre maternité entre le 16 et 28 novembre 2017, pendant une période de 12 jours consécutifs. Un questionnaire écrit leur était proposé pendant leur séjour du post-partum. II était court et comportait 15 items répartis sur une double page, avec deux questions ouvertes pour permettre aux mères de s'exprimer librement. Toutes les patientes ont donné leur accord oral. L'étude a fait l'objet d'une déclaration CNIL (DEC16-401).

Dans notre maternité, environ $18 \%$ des patientes accouchent par césarienne, dont environ la moitié de manière programmée. Dès la décision, toutes les césariennes sont classées selon un code couleur en trois classes selon leur niveau d'urgence [19] : le délai entre la décision de césarienne et l'extraction du nouveau-né doit être inférieur à 15 minutes en cas de code rouge, 30 minutes en cas de code orange, et 60 minutes en cas de code vert. II existe en permanence une équipe de garde avec anesthésiste, pédiatre et gynécologue-obstétricien présents sur place. 
Les données ont été enregistrées et analysées avec le logiciel Epi-Data software (version 3.1, Epidata Association, Danemark). Les pourcentages figurent entre parenthèses. Les comparaisons entre pourcentages ont fait appel au test du Chi2, et au test de Fisher en cas d'effectifs faibles. Les différences étaient considérées comme significatives si $p<0.05$. 


\section{Résultats}

185 patientes ont accouché pendant la période d'étude, dont 35 patientes par césarienne (19\%). Les conditions de réalisation de la césarienne sont présentées au Tableau 1. Parmi elles, les trois quarts étaient non programmées et un quart concernaient des enfants prématurés. Environ la moitié des césariennes ont eu lieu en code vert, un tiers en code orange et $14 \%$ en code rouge. L'analgésie était locorégionale dans la grande majorité des cas. Parmi les 26 conjoints présents dans la maternité au moment de la césarienne, 24 se sont vu proposer d'assister à la césarienne et tous ont accepté (92\%).

Le peau-à-peau a été pratiqué dans un peu moins de la moitié des césariennes (Tableau 2). II était presque systématique en cas de césarienne programmée mais n'a eu lieu que dans un tiers des césariennes non programmées pendant la même période (89 vs 31\%, p<0.005). De la même façon, la pratique du peau-à-peau était également beaucoup plus fréquente en cas de code vert (72\%) qu'en cas de code orange ou rouge (25 et $0 \%$, respectivement, $p<0.001$ ). En pratique lorsqu'il était réalisé, le peau-à-peau débutait le plus souvent dans les 5 minutes qui suivaient l'extraction du nouveau-né. Il n'a pu être maintenu pendant toute la durée de la césarienne que dans un quart des cas $(n=4)$. Parmi les 19 césariennes dans lesquelles le peau-à-peau n'a pas été réalisé avec la mère, il s'agissait de contreindications maternelles dans deux tiers des cas, principalement du fait d'un état de conscience ou de disponibilité insuffisant (malaise, hémorragie, douleur ressentie...) et de contre-indications néonatales dans presque tous les autres cas (prématurité inférieure à $34 \mathrm{SA}, \mathrm{pH}$ artériel $<7.05$ au cordon ombilical). Dans ces conditions, un 
peau-à-peau avec le père a été réalisé dans la majorité des 12 cas où il était présent (83\%), et il a pu être pratiqué en salle de réveil avec la mère dans $82 \%$ des cas. A noter que dans le groupe des césariennes programmées (9 patientes), la seule patiente n'ayant pas pu avoir de peau à peau était sous anesthésie générale pour inefficacité de la péridurale. 


\section{Discussion}

Notre étude montre que la réalisation d'un peau-à-peau est possible au décours d'une césarienne, ce qui est en accord avec les études réalisées depuis une dizaine d'années sur ce sujet $[12,14]$. Elle indique également que le peau a peau n'a été possible que dans la moitié des césariennes (46\%), et ce taux varie globalement de 30 à $80 \%$ dans la littérature [12-14]. A titre d'exemple aux Etats-Unis, le taux de peau-à-peau en cas de césarienne était seulement de $30 \%$ parmi les enfants nés dans une grande structure de type III universitaire [13], mais atteignait $74 \%$ dans un autre centre du même type [14].

Les raisons de cette hétérogénéité ne sont pas toutes connues mais il est probable que la motivation des équipes joue un rôle ; les chiffres les plus élevés pourraient aussi être observés dans les centres qui ont mis en place le peau à peau depuis plus longtemps, avec une ascension progressive des taux [13]. II est possible enfin que les centres ayant des taux élevés d'accouchement par césarienne puissent avoir un pourcentage élevé de peau à peau, du fait d'un taux élevé de césariennes programmées [12-14]. Notre étude a en effet montré que le peau à peau est nettement plus fréquent en cas de programmation. Des résultats superposables aux nôtres sont retrouvés par une équipe espagnole d'une maternité universitaire qui observe un taux de peau à peau de $44 \%$, variant de $80 \%$ en cas de césarienne programmée à seulement $29 \%$ en cas de césarienne non programmée [20]. Dans notre série, l'absence de peau à peau était principalement liée à l'existence de contre-indications maternelles, bien plus fréquentes en cas de césariennes non 
programmées: absence de conscience ou de disponibilité maternelle (émotion, stress, douleurs, nausées, sensation de malaise et/ou perte de conscience).

Dans notre étude, presque tous les nouveau-nés mis en peau à peau l'ont été dans les 5 minutes qui suivaient son extraction, mais seulement un quart ont été maintenus en peau à peau pendant toute la durée de la césarienne. II est vraisemblable que les interruptions de peau à peau ont eu les mêmes causes que l'absence de peau à peau, mais ces éléments ne figuraient pas dans notre recueil de données. Limiter ces interruptions en cas de césarienne nous parait aussi important qu'en cas de voie basse, puisqu'elles retardent l'initiation de l'allaitement [21].

En cas d'impossibilité de peau à peau maternel, $83 \%$ des conjoints de notre série ont réalisé du peau à peau avec le nouveau-né, ce qui est satisfaisant. II a en effet été montré que le conjoint peut réchauffer le nouveau-né aussi efficacement qu'un incubateur, avec des taux de glycémie significativement supérieurs à ceux des enfants séparés ou mis en berceau [22]. Lors du peau à peau paternel, on observe également une diminution significative des cris et un endormissement plus rapide [23]. Les mouvements de tétée apparaissent plus tôt que chez les enfants mis en berceau [23]. Du point de vue de l'attachement, des auteurs suédois montrent qu'en cas de peau à peau le père communique verbalement plus avec son enfant, et qu'il communique également significativement plus avec la mère [24]. Enfin, dans notre série, la réalisation de peau à peau en salle de réveil était pratiquement réalisée dans tous les cas où l'état des nouveau-nés le permettait, ce qui est conforme aux recommandations internationales [25]. 
Finalement, notre étude d'évaluation des pratiques montre que le peau à peau maternel est possible en cas de césarienne, avec beaucoup de bénéfices maternels, paternels et néonataux $[14,23,26]$. Bien que la température soit abaissée dans les salles de bloc opératoire et que les interactions mère-bébé puissent être modifiés par l'anesthésie [8,27], on assiste à une réduction significative des pleurs dès le premier quart d'heure [16,28], un endormissement plus rapide [23], une meilleure stabilité thermique [15]. Une étude d'anesthésiologie rappelle l'intérêt de l'utilisation d'une couverture chauffante tant que la patiente est au bloc [29]. Si la mère en ressent le besoin, une couverture chauffante type « haut-de-corps » peut d'ailleurs être placée transversalement en cours de césarienne. II existe également une diminution du risque d'hypoglycémie par économie sur le plan métabolique [26], ainsi qu'une réduction par deux à trois du taux de transfert en soins intensifs néonataux (OR 0.58, $95 \% \mathrm{Cl}$ 0.41-0.80) [30,31]. La fréquence des antibiothérapies pour suspicion d'infection néonatale précoce est également réduite(OR $0.4, \mathrm{Cl} 95 \%, 0.19-0.83$ ) [31]. Chez le prématuré, le peau à peau pourrait s'accompagner d'un effet protecteur vis-à-vis des infections nosocomiales graves [3], la flore cutanée de la mère permettant la maturation de la flore digestive du nouveau-né et favorisant probablement son développement immunitaire [32]. En ce qui concerne la mère, le peau-à peau ne s'accompagne pas d'augmentation des infections de site opératoire [31].

En ce qui concerne les mères et la relation mère-enfant, les patientes en peau à peau nécessitent moins d'antalgiques pendant la césarienne [33]. Les parents ont l'impression d'une durée de césarienne plus courte et perçoivent les soins comme meilleurs, que ce soient ceux des sages-femmes, des obstétriciens, des infirmières 
ou des anesthésistes [34]. Les mères font par ailleurs état du sentiment d'avoir bien fait pour leur bébé, et ont davantage confiance en leurs capacités parentales [35]. Les taux d'allaitement à la sortie sont plus élevés [13,36]. Les patientes bénéficiant pour la première fois du peau à peau après une ou plusieurs césariennes suivies de séparation sont plus satisfaites de la dernière césarienne, et expriment moins d'anxiété vis-à-vis de la santé et du bien-être de leur bébé [20,37]. Les scores de dépression du post-partum pourraient être améliorés [5].

Malgré ses nombreux avantages enfin, il est malheureusement primordial de rappeler que le peau à peau s'accompagne parfois d'authentiques collapsus néonataux - quelquefois mortels - pendant les deux premières heures de vie, même chez des enfants sans facteur de risque. Leur fréquence varie entre 3.2 et 27 pour 100000 chez le nouveau-né à terme [38,39]. Dans ces conditions, des précautions s'imposent en salle de naissance, pour une césarienne comme pour une voie basse : mère en position redressée, nez et bouche du bébé dégagés, tête tournée sur le côté, surveillance constante par le personnel soignant ou, si la surveillance est impossible, monitorage de la saturation et de la fréquence cardiaque du bébé [18].

En définitive, cette étude nous encourage à diffuser le peau à peau en cours de césarienne, pratique qui nous paraissait presque impossible lorsque nous avons débuté... alors qu'elle est largement répandue dans les pays scandinaves et anglosaxons, avec une utilité démontrée [2,3,40]. Dans notre expérience, la crainte d'une réduction de l'espace opératoire est injustifiée, l'intervention étant sous-ombilicale et le nouveau-né placé transversalement sur la poitrine maternelle, sans gêne pour l'opérateur ni son aide. Les obstétriciens qui réalisent des césariennes dans notre 
pays - notamment lorsqu'elles sont programmées - devraient connaître cette possibilité qui améliore la satisfaction des patientes et possède de nombreux avantages à court et à long terme. 


\section{Remerciements}

Les auteurs remercient Alia ALAMI, Alice BOUTEZ, Florine LEFEBVRE, Marion LESAGE, Louise MARTINET et Jessie PHANOUVONG qui ont accepté de concevoir le questionnaire et de mener l'enquête auprès des patientes de l'étude.

\section{Conflits d'intérêt}

Les auteurs déclarent ne pas avoir de liens d'intérêts. 


\section{Références}

1. OMS, UNICEF : «Déclaration conjointe : Protection, encouragement et soutien de l'allaitement maternel. Le rôle spécial des services liés à la maternité » OMS, Genève, 1989 [Internet]. Available from: http://apps.who.int/iris/bitstream/10665/39875/1/9242561304_fre.pdf

2. Moore ER, Bergman N, Anderson GC, Medley N. Early skin-to-skin contact for mothers and their healthy newborn infants. Cochrane Database Syst Rev. 2016 25;11:CD003519.

3. Conde-Agudelo A, Díaz-Rossello JL. Kangaroo mother care to reduce morbidity and mortality in low birthweight infants. Cochrane Database Syst Rev. 2016:CD002771.

4. Bowlby J. Attachement et perte. Vol1, L'attachement. 2002. Presses Universitaires de France.

5. Feldman R, Eidelman AI, Sirota L, Weller A. Comparison of Skin-to-Skin (Kangaroo) and Traditional Care: Parenting Outcomes and Preterm Infant Development. Pediatrics. 2002;110:16-26.

6. Zwedberg S, Blomquist J, Sigerstad E. Midwives' experiences with mother-infant skin-toskin contact after a caesarean section: "fighting an uphill battle." Midwifery. 2015;31:215-20.

7. Rowe-Murray HJ, Fisher JRW. Baby friendly hospital practices: cesarean section is a persistent barrier to early initiation of breastfeeding. Birth Berkeley Calif. 2002;29:12431.

8. DiMatteo MR, Morton SC, Lepper HS, Damush TM, Carney MF, Pearson M, et al. Cesarean childbirth and psychosocial outcomes: a meta-analysis. Health Psychol. 1996;15:303-14.

9. Chen C, Yan Y, Gao X, Xiang S, He Q, et al. Effects of Cesarean Delivery on Breastfeeding Practices and Duration: A Prospective Cohort Study. J Hum Lact 2018 34:526-534.

10. Porter M, van Teijlingen E, Chi Ying Yip L, Bhattacharya S. Satisfaction with cesarean section: qualitative analysis of open-ended questions in a large postal survey. Birth. 2007;34:148-54.

11. Smith J, Plaat F, Fisk NM. The natural caesarean: a woman-centred technique. BJOG Int J Obstet Gynaecol. 2008;115:1037-42.

12. Grassley JS, Jones J. Implementing skin-to-skin contact in the operating room following cesarean birth. Worldviews Evid Based Nurs. 2014;11:414-6.

13. Schorn MN, Moore E, Spetalnick BM, Morad A. Implementing Family-Centered Cesarean Birth. J Midwifery Womens Health. 2015;60:682-90.

14. Boyd MM. Implementing Skin-to-Skin Contact for Cesarean Birth. AORN J. 2017;105:579-92. 
15. Kollmann M, Aldrian L, Scheuchenegger A, Mautner E, Herzog SA, et al. Early skin-toskin contact after cesarean section: A randomized clinical pilot study. PloS One. 2017;12:e0168783.

16. Gray L, Watt L, Blass EM. Skin-to-skin contact is analgesic in healthy newborns. Pediatrics. 2000;105:e14.

17. Ducloy-Bouthors AS, Cordier D, Lemort L, Dalmas-Laurent AF, Detailleur C et al. Peau à peau en salle de césarienne. Rev Med Perinat. 2018 ;10 :125-6.

18. Branger B. Indications et surveillance du nouveau-né à terme en peau-à-peau en salle de naissance [Internet]. 2013. Available from:

file:///C:/Users/patri/AppData/Roaming/Zotero/Zotero/Profiles/bmnq0jvc.default/zotero/st orage/ASRMA4VF/8xvxtqj46v4ddpwtyidq5jfhja3644-org.pdf

19. Huissoud C, du Mesnildot P, Sayegh I, Dupuis O, Clément H-J, et al. La mise en œuvre des codes « couleur » réduit le délai décision-naissance des césariennes urgentes. J Gynecol Obstet Biol Reprod.2009;38:51-9.

20. de Alba-Romero C, Camaño-Gutiérrez I, López-Hernández P, de Castro-Fernández J, Barbero-Casado P, et al. Postcesarean Section Skin-to-Skin Contact of Mother and Child. J Hum Lact. 2014;30:283-6.

21. Robiquet P, Zamiara P-E, Rakza T, Deruelle P, Mestdagh B, et al. Observation of Skinto-Skin Contact and Analysis of Factors Linked to Failure to Breastfeed Within 2 Hours After Birth. Breastfeed Med. 2016;11:126-32.

22. Christensson K. Fathers can effectively achieve heat conservation in healthy newborn infants. Acta Paediatr. 1996;85:1354-60.

23. Erlandsson K, Dsilna A, Fagerberg I, Christensson K. Skin-to-skin care with the father after cesarean birth and its effect on newborn crying and prefeeding behavior. Birth. 2007;34:105-14.

24. Velandia M, Matthisen A-S, Uvnäs-Moberg K, Nissen E. Onset of vocal interaction between parents and newborns in skin-to-skin contact immediately after elective cesarean section. Birth. 2010;37:192-201.

25. WHO | Kangaroo mother care: a practical guide [Internet]. WHO. [cited 2018 May 26]. Available from: http://www.who.int/maternal_child_adolescent/documents/9241590351/en/

26. LeBlanc S, Haushalter J, Seashore C, Wood KS, Steiner MJ, et al. A QualityImprovement Initiative to Reduce NICU Transfers for Neonates at Risk for Hypoglycemia. Pediatrics. 2018 ;141, e20171143.

27. Christensson K, Siles C, Cabrera T, Belaustequi A, de la Fuente P, et al. Lower body temperatures in infants delivered by caesarean section than in vaginally delivered infants. Acta Paediatr. 1993;82:128-31.

28. Michelsson K, Christensson K, Rothgänger H, Winberg J. Crying in separated and nonseparated newborns: sound spectrographic analysis. Acta Paediatr. 1996;:471-5. 
29. Horn E-P, Bein B, Steinfath M, Ramaker K, Buchloh B, et al. The incidence and prevention of hypothermia in newborn bonding after cesarean delivery: a randomized controlled trial. Anesth Analg. 2014;118:997-1002.

30. Schneider LW, Crenshaw JT, Gilder RE. Influence of Immediate Skin-to-Skin Contact During Cesarean Surgery on Rate of Transfer of Newborns to NICU for Observation. Nurs Womens Health. 2017;21:28-33.

31. Posthuma S, Korteweg FJ, van der Ploeg JM, de Boer HD, Buiter HD, et al. Risks and benefits of the skin-to-skin cesarean section - a retrospective cohort study. J Matern-Fetal Neonatal Med. 2017;30:159-63.

32. Lamy Filho F, de Sousa SHC, Freitas IJS, Lamy ZC, Simões VMF, et al. Effect of maternal skin-to-skin contact on decolonization of Methicillin-Oxacillin-Resistant Staphylococcus in neonatal intensive care units: a randomized controlled trial. BMC Pregnancy Childbirth. 2015;15:63.

33. Sundin CS, Mazac LB. Implementing Skin-to-Skin Care in the Operating Room After Cesarean Birth. MCN Am J Matern Child Nurs. 2015;40:249-55.

34. Armbrust R, Hinkson L, von Weizsäcker K, Henrich W. The Charité cesarean birth: a family orientated approach of cesarean section. J Matern-Fetal Neonatal Med. 2016;29:163-8.

35. Tessier R, Cristo M, Velez S, Giron M, de Calume ZF, et al. Kangaroo mother care and the bonding hypothesis. Pediatrics. 1998;(2):e17.

36. Brady K, Bulpitt D, Chiarelli C. An interprofessional quality improvement project to implement maternal/infant skin-to-skin contact during cesarean delivery. J Obstet Gynecol Neonatal Nurs. 2014;43:488-96.

37. Moran-Peters JA, Zauderer CR, Goldman S, Baierlein J, Smith AE. A quality improvement project focused on women's perceptions of skin-to-skin contact after cesarean birth. Nurs Womens Health. 2014;18:294-303.

38. Dageville C, Pignol J, De Smet S. Very early neonatal apparent life-threatening events and sudden unexpected deaths: incidence and risk factors. Acta Paediatr. 2008;97:866-9.

39. Rodríguez-Alarcón J, Melchor JC, Linares A, Aranguren G, Quintanilla M, et al. Early neonatal sudden death or near death syndrome. An epidemiological study of 29 cases. Acta Paediatr. 1994;83:704-8.

40. Nyqvist KH, Expert Group of the International Network on Kangaroo Mother Care, Anderson GC, Bergman N, Cattaneo A, et al. State of the art and recommendations. Kangaroo mother care: application in a high-tech environment. Acta Paediatr. 2010;8129. 
Figure 1. Peau à peau en cours de césarienne

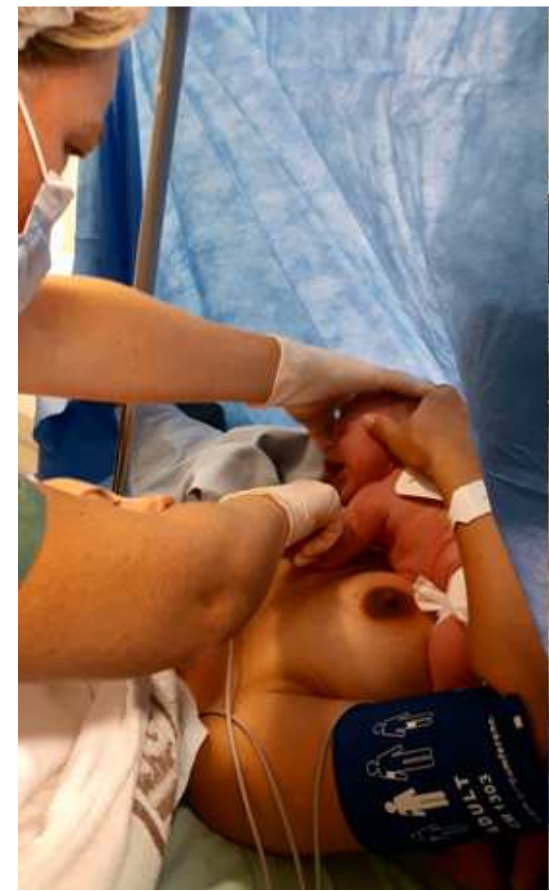

1

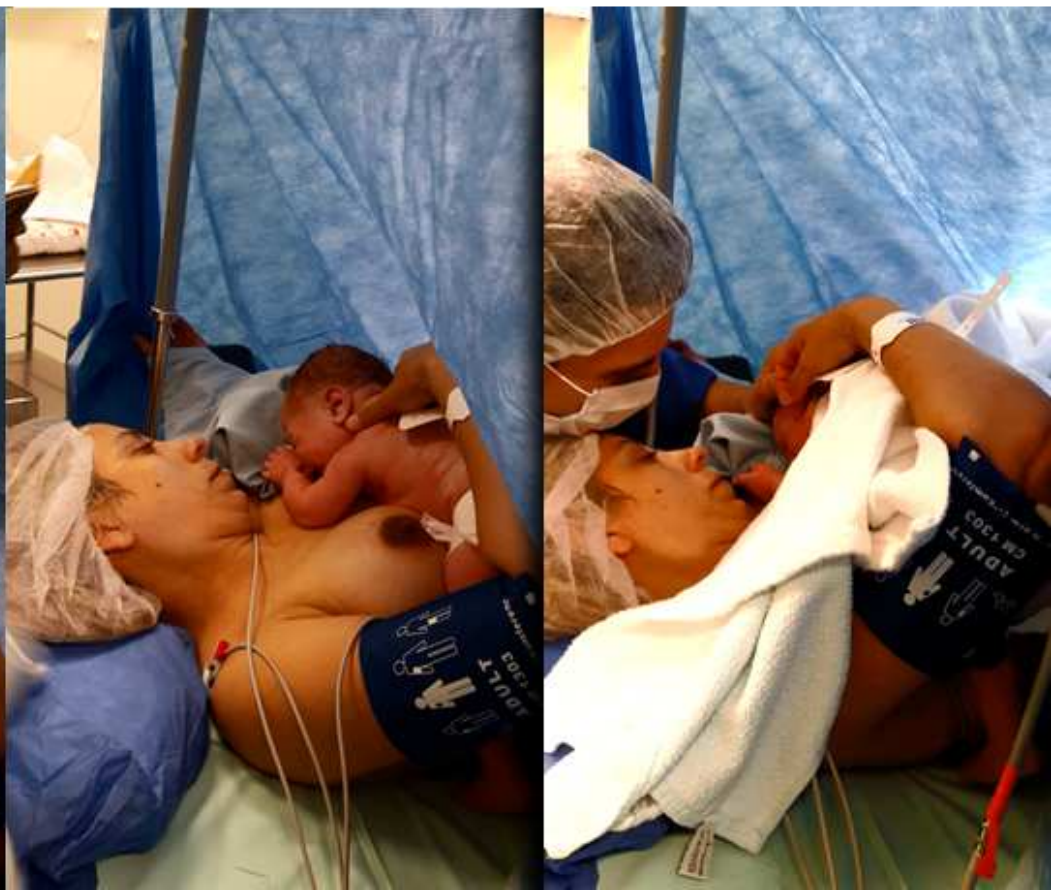

2
3

1. La sage-femme installe le nouveau-né sur sa mère en dégageant son visage. Le conjoint s'est écarté pour la laisser passer.

2. La position du nouveau-né est stable malgré l'absence - sur cette photo - de jersey enfilé par la patiente préalablement à la césarienne, qui l'aurait maintenu de façon optimale. Ne pas oublier ce jersey en cas d'urgence serait idéal.

3. Le nouveau-né est recouvert d'une couverture ; le conjoint a repris sa place. En cas de besoin de la patiente, une couverture chauffante type " haut-decorps " peut être mise en place au-dessus du nouveau-né, qui reste sous le regard de sa mère. 
Tableau 1. Conditions de réalisation de la césarienne

$n=35$

Césarienne non programmée

$26(74)$

Césarienne avant 37 SA

$9(26)$

Code couleur de la césarienne

Vert

$18(51)$

Orange $(<30 \mathrm{~min})$

$12(34)$

Rouge $(<15 \mathrm{~min})$

$5(14)$

Anesthésie

Loco-régionale

$32(91)$

Générale

$3(8)$

Proposition au conjoint d'assister à la césarienne 
Tableau 2. Peau à peau en cours de césarienne

$\mathrm{n}=35$

$\mathrm{p}$

Peau à peau en cours de césarienne $16(46)$

Peau à peau selon le type de césarienne

Programmée

$8 / 9(89)$

Non programmée

$8 / 26(31)<0.005$

Peau à peau selon le code couleur de la césarienne*

Vert

$13 / 18(72)$

Orange

$3 / 12(25) \quad<0.001$

Rouge

$0 / 5(0)$

Durée du peau à peau pendant la césarienne $(n=16)$

Dans les 5 minutes

Maintenu pendant toute la césarienne

$4 / 16(25)$

Raisons de l'absence de peau à peau pendant la césarienne $(\mathrm{n}=19)$

Contre-indications **

$18 / 19(95)$

Néonatale 5

Maternelle 13

Refus maternel $1 / 19(5)$

Si impossibilité, réalisation du peau à peau avec le père $10 / 12(83)^{\star \star *}$

Peau à peau en salle de réveil 27/33 (82)

* Le test a comparé les codes verts aux codes orange et rouge rassemblés

** Voir Matériel et Méthodes

*** Seulement 12 conjoints parmi 19 étaient présents dans les situations où le peau à peau était impossible 